Bull. Austral. Math. Soc.

VOL. 52 (1995) [313-315]

\title{
ON THE SPLITTING FIELD OF THE ALEXANDER POLYNOMIAL OF A PERIODIC KNOT
}

\author{
Jonathan A. Hillman
}

We show that the Murasugi conditions for the Alexander polynomial of a cyclically periodic knat imply a modified form of the Burde-Trotter condition.

Let $K$ be a knot in $S^{3}$ with Alexander polynomial $\Delta_{K}(t)$ and which is invariant under a rotation of prime power order $q=p^{r}$ about a disjoint axis $A$. Let $\bar{K}$ and $\bar{A}$ be the images of $K$ and $A$ in the orbit space $S^{3} /(Z / q Z) \cong S^{3}$. Murasugi showed that $\Delta_{K}(t)=\Delta_{\bar{K}}(t) \prod_{i=1}^{q-1} f\left(t, \zeta_{q}^{i}\right)$, where $f(t, u)$ is the 2-variable Alexander polynomial of $\bar{K} \cup \bar{A}$ and $\zeta_{q}$ is a primitive $q$-th root of unity [7]. In particular, $f\left(t, \zeta_{q}^{i}\right)$ is congruent modulo $(p)$ to $f(t, 1)=\left(\left(t^{\lambda}-1\right) /(t-1)\right) \Delta_{\bar{K}}(t)$, where $\lambda=\operatorname{link}(K, A)=\operatorname{link}(K, \bar{A})$. On reducing the equation $\Delta_{K}(t)=\Delta_{\bar{K}}(t) \prod_{\sigma \in G} f(t)^{\sigma}$ modulo $(t-1, p)$ we get $1=\lambda^{q-1}$ modulo $(p)$, and so $(\lambda, p)=1$. (This is also clear for topological reasons, since $K$ is connected.) If moreover $\Delta_{K}(t) \equiv 1$ modulo $(p)$ then $\lambda=1$.

Trotter showed that if the commutator subgroup of the knot group is free and if $\Delta_{K}$ has no repeated roots then the $q$-th roots of unity are in $\operatorname{Split}\left(\Delta_{K} / Q\right)$, the splitting field of $\Delta_{K}$ over $Q$ [8]. Burde weakened these hypotheses to requiring that $\Delta_{K}(t) \not \equiv 1$ modulo $(p)$ and that the second Alexander polynomial $\Delta_{2, K}(t)$ be trivial [1]. This was extended to all knots as a condition involving the higher Alexander polynomials: if $\Delta_{K}(t) \not \equiv 1$ modulo $(p)$ but $\Delta_{n+1, K}(t)=1$ then $\zeta_{q}$ has degree at most $n$ over $\operatorname{Split}\left(\Delta_{K} / Q\right)$ [6]. The higher Alexander polynomials are determined by (and determine) the structure of $H_{1}\left(X^{\prime} ; Q\right)$, the first homology group with rational coefficients of the infinite cyclic cover of the knot complement, as a module over the principal ideal domain $Q\left[t, t^{-1}\right]$. It follows easily from the elementary divisor theorem (that is, essentially the structure theorem for finitely generated modules over a PID) that $\Delta_{n+1, K}(t)=1$ if $\Delta_{K}$ has no irreducible factor of multiplicity greater than $n$. Hence the Burde-Trotter condition implies that $\left[Q\left(\zeta_{q}\right): Q\left(\zeta_{q}\right) \cap S p l i t\left(\Delta_{K} / Q\right)\right] \leqslant m$, where $m$ is the maximal multiplicity of irreducible factors of $\Delta_{K}$. This condition involves only $\Delta_{K}$ and is closer to Trotter's original formulation.

Received 6th December, 1995

Copyright Clearance Centre, Inc. Serial-fee code: 0004-9729/95 \$A2.00+0.00. 
In this note we shall show that the Murasugi conditions as stated above imply this modified form of the Burde-Trotter condition. The Burde-Trotter and Murasugi conditions have been extended to knots in homology 3-spheres [5], and the present argument applies equally well to such knots. (Note that the formulation of the Murasugi conditions in [3] assumes that the Alexander polynomial of the axis $\bar{A}$ is trivial, as is always the case when the homology spheres involved are both $S^{3}$.) We also give simple examples to show that the Murasugi conditions do not imply the full BurdeTrotter condition involving the higher Alexander polynomials, and that the congruence alternative is needed in general.

THEOREM. Let $\Delta$ be a polynomial in $Z[t]$ which satisfies the Murasugi conditions for some prime power $q=p^{r}$. Suppose $\Delta$ has irreducible factorisation $\Delta=\prod \delta_{i}^{e_{i}}$. Then either $\Delta \equiv 1$ modulo $(p)$ or $\left[Q\left(\zeta_{q}\right): Q\left(\zeta_{q}\right) \cap S p l i t(\Delta / Q)\right] \leqslant m=\max \left\{e_{i}\right\}$.

Proof: Let $G=\operatorname{Gal}\left(Q\left(\zeta_{q}\right) / Q\right)$. The Murasugi conditions assert that there is an integer $\lambda$, a knot polynomial $\widehat{\Delta}$ and a polynomial $f(t)$ in $Z\left(\zeta_{q}\right)[t]$ such that $\Delta=$ $\widehat{\Delta} \prod_{\sigma \in G} f^{\sigma}$ and $f(t) \equiv\left(\left(t^{\lambda}-1\right) /(t-1)\right) \widehat{\Delta}$ modulo $(p)$. We may assume that $\Delta \neq \equiv 1$ modulo $(p)$. Then $f$ is nontrivial and so has a nontrivial irreducible factor $h$ in $Q\left(\zeta_{q}\right)[t]$. Let $S=\left\{\sigma \in G \mid h^{\sigma}=h\right\}$, and let $T$ be a set of coset representatives for $S$ in $G$. Then $H=\prod_{\tau \in T} h^{\tau}$ is irreducible in $Z[t]$ and $H^{|S|}=\prod_{\sigma \in G} h^{\sigma}$ divides $\Delta$, so $|S| \leqslant m$. Let $M=Q\left(\zeta_{q}\right)^{S}$ be the subfield of $Q\left(\zeta_{q}\right)$ fixed by $S$. Then $M$ is generated over $Q$ by the coefficients of $h$, and $\left[Q\left(\zeta_{q}\right): M\right]=|S|$. Since the coefficients of $h$ are elementary symmetric functions of the roots of $h$, which are among the roots of $\Delta$, they are in $\operatorname{Split}(\Delta / Q)$. The theorem follows easily.

Let $f(t)=t^{2}-3 t+1$ be the Alexander polynomial of the figure eight knot. Then $\Delta=f^{3}$ satisfies the Murasugi conditions with $n=3$ and $\lambda=1$. It is the Alexander polynomial of the connected sum of three copies of the figure eight knot, which admits an obvious $Z / 3 Z$ symmetry. However the full Burde-Trotter condition implies that no knot with this Alexander polynomial and which admits such a cyclic symmetry can have a cyclic knot module. (In particular, no such knot can have unknotting number 1.) The polynomial $3 t^{2}-5 t+3$ satisfies the Murasugi conditions with $q=3$ and $\lambda=1$, and hence is the Alexander polynomial of some knot with cyclic period 3 , by Theorem 1.1 of [3]. As the splitting field for this quadratic is $Q(\sqrt{-11})$, which does not contain $Q\left(\zeta_{3}\right)$, we see that in general the alternative " $\Delta_{K}(t) \equiv 1 \operatorname{modulo}(p)$ " is necessary. (This answers a question raised in the "Remark" on page 266 of [2].)

\section{REFERENCES}

[1] G. Burde, 'Uber periodische Knoten', Arch. Math. (Basel) 30 (1978), 487-492. 
[2] G. Burde and H. Zieschang, Knots, Studies in Mathematics 5 (W. de Gruyter, Berlin, New York, 1985).

[3] J.M. Davis and C. Livingston, 'Alexander polynomials of periodic knots', Topology 30 (1991); 551-564.

[4] J.A. Hillman, Alexander Ideals of Links, Lecture Notes in Mathematics 895 (Springer-Verlag, Berlin, Heidelberg, New York, 1981).

[5] J.A. Hillman, 'New proofs of two theorems on periodic knots', Arch. Math. (Basel) 37 (1981), 457-461.

[6] J.A. Hillman, 'On the Alexander polynomial of a cyclically periodic knot', Proc. Amer. Math. Soc. 89 (1983), 155-156.

[7] K. Murasugi, 'On periodic knots', Comment. Math. Helv. 46 (1971), 162-174.

[8] H.F. Trotter, 'Periodic automorphisms of groups and knots', Duke Math. J. 28 (1961), 553-557.

School of Mathematics and Statistics

The University of Sydney

New South Wales 2006

Australia 